\title{
Dynamics and invariant manifolds for a nonlocal stochastic Swift-Hohenberg equation
}

\section{Yanfeng Guo*}

\section{"Correspondence:}

guoyan_feng@163.com

College of Science, Guangxi

University of Science and

Technology, Liuzhou, Guangxi

545006, P.R. China

\section{照 Springer}

\begin{abstract}
The dynamics and invariant manifolds for a nonlocal stochastic Swift-Hohenberg equation with multiplicative noise are investigated. Using a stochastic transformation process, a nonlocal stochastic Swift-Hohenberg equation is studied with either a positive kernel or a non-negative kernel. Then the dynamics, existence, and uniqueness of a global random attractor for the nonlocal stochastic Swift-Hohenberg equation is shown. Moreover, the existence of a local random invariant manifold of the corresponding random dynamical system for the nonlocal stochastic Swift-Hohenberg equation with multiplicative noise is obtained using the technique of a cut-off function and the Lyapunov-Perron method.
\end{abstract}

MSC: $60 \mathrm{H} 15 ; 76 \mathrm{~A} 05 ; 37 \mathrm{H} 05 ; 37 \mathrm{~L} 55$

Keywords: nonlocal stochastic Swift-Hohenberg equation; stochastic transformation; cut-off function; random attractor; invariant manifolds

\section{Introduction}

It is well known that fluid convection due to density gradients arises in geophysical fluid flows in the atmosphere, oceans, and the earth's mantle. Rayleigh-Bénard convection is a prototypical model for fluid convection in predicting spatio-temporal convection patterns. It is very important that the mathematical model for the Rayleigh-Bénard convection is closely contacted with nonlinear Navier-Stokes equations coupled with the temperature equation. When the Rayleigh number is near the onset of the convection, the Rayleigh-Bénard convection model may be approximately reduced to an amplitude or order parameter equation [1]. We know that a localized one-dimensional version of the model is the local one-dimensional Swift-Hohenberg equation

$$
u_{t}=-\left(1+\partial_{x x}\right)^{2} u+\mu u-u^{3}
$$

which was originally derived by Swift and Hohenberg [1] as a model for the convective instability in the Rayleigh-Bénard convection. In addition, the Swift-Hohenberg equation was not only derived for one-dimensional models, furthermore, it is also well studied in high-dimensional models. We know there has been some research recently as regards the local Swift-Hohenberg equation [2-5].

(c) 2015 Guo. This article is distributed under the terms of the Creative Commons Attribution 4.0 International License (http://creativecommons.org/licenses/by/4.0/), which permits unrestricted use, distribution, and reproduction in any medium, provided you give appropriate credit to the original author(s) and the source, provide a link to the Creative Commons license, and indicate if changes were made. 
However, when the distance from the change of stability is sufficiently small, or the Rayleigh number is near thermal equilibrium, the influence of small noise or molecular noise is detected in various convection experiments [6-8]. It is difficult to stabilize the control parameters (e.g. the temperature in Rayleigh-Bénard convection) to the precision of the noise strength, which is extremely small in the case of thermal fluctuations. When the effects of thermal fluctuations (i.e. additive noise) on the onset of convective motion are considered in the Bénard system, a local stochastic Swift-Hohenberg equation with additive noise [1] is proposed:

$$
u_{t}=-\left(1+\partial_{x x}\right)^{2} u+\mu u-u^{3}+\sigma \xi
$$

Furthermore, it is also allowed to consider the effects of small possible noise from $\mu$. So a local stochastic Swift-Hohenberg equation with multiplicative noise[9] arises,

$$
u_{t}=-\left(1+\partial_{x x}\right)^{2} u+\mu u-u^{3}+\sigma u \circ \xi
$$

Here $\sigma>0$ and $\xi=\frac{d W}{d t}$ is the generalized derivative of a real valued one-dimensional Brownian motion $W(t)$. We know that the cubic term $u^{3}$ is used as an approximation of a nonlocal integral term [2, 3]. In the classical paper [1] by Swift and Hohenberg the analysis already hints toward a nonlocal nonlinearity. It is only for simplicity that they just assumed the standard cubic. Recently, Roberts $[10,11]$ reexamined the rationale for using the Swift-Hohenberg model as the reliable model of the spatial pattern evolution in a specific physical system. He notes that, although the localization approximation used in (1.1) makes some sense in the one-dimensional case, this approximation is deficient in the two-dimensional convection problem $[1,10,11]$ and one should use the nonlocal SwiftHohenberg model

$$
u_{t}=-(1+\Delta)^{2} u+\mu u-u G * u^{2}
$$

where

$$
G * u^{2}=\int_{D} G\left(\sqrt{(x-\xi)^{2}+(y-\eta)^{2}}\right) u^{2}(\xi, \eta, t) d \xi d \eta,
$$

and $u(x)$ is the unknown amplitude function, $\mu$ measures the difference of the Rayleigh number from its critical onset value, $\Delta=\partial_{x x}+\partial_{y y}$ is the Laplace operator with respect to the variable $x \in \mathbb{R}^{2}, G(r)\left(r=\sqrt{x^{2}+y^{2}}\right)$ is a given radially symmetric function, and $*$ denotes the convolution. The equation is defined for $t>0$ and $x \in D$, where $D$ is a bounded planar domain with smooth boundary $\partial D$. When one considers the smallness of the fluctuation effects as for the local Swift-Hohenberg equation, the two-dimensional version of nonlocal stochastic Swift-Hohenberg equation [9] can be written as

$$
u_{t}=-(1+\Delta)^{2} u+\mu u-u G * u^{2}+\sigma u \circ \xi
$$

By a similar consideration, the two-dimensional version of the local stochastic SwiftHohenberg equation for $u(x, y, t)$ can be written as

$$
u_{t}=-(1+\Delta)^{2} u+\mu u-u^{3}+\sigma u \circ \xi .
$$


But the nonlocal stochastic Swift-Hohenberg equation (1.6) has had little investigation so far [9]. Since the nonlocal equations are more significant to explain the nonlinear physical phenomenon, it is important to investigate the dynamics behaviors for them. Therefore, against the stronger background of the nonlocal stochastic Swift-Hohenberg equation, there is new work to consider it in present paper. The purpose of this paper is mainly to consider the nonlocal stochastic Swift-Hohenberg equation with positive kernel and non-negative kernel. The global random attractor and existence of invariant manifolds are investigated for the two-dimensional nonlocal stochastic Swift-Hohenberg equation with multiplicative noise defined on a bounded planar domain $D$ in $\mathbb{R}^{d}(d=2)$. Furthermore, for the three-dimensional case, the parallel results have been obtained by a similar procedure. Therefore, in this paper, we mainly investigate it in the two-dimensional case. The problem has been written as follows:

$$
d u=-(1+\Delta)^{2} u d t+\mu u d t-u G * u^{2} d t+\sigma u \circ d W(t)
$$

with initial condition

$$
u(x, y, 0)=u_{0}(x, y), \quad(x, y) \in D
$$

and the boundary conditions

$$
\left.u\right|_{\partial D}=\left.\frac{\partial u}{\partial n}\right|_{\partial D}=0, \quad(x, y) \in \partial D
$$

Specifically, using a stochastic transformation and the technique of a cut-off function, the nonlocal stochastic Swift-Hohenberg equation is studied with either a positive kernel or a non-negative kernel. Then the existence and uniqueness of a global random attractor for the nonlocal stochastic Swift-Hohenberg equation is shown. Moreover, the existence of a local random invariant manifold of the corresponding random dynamical system for the nonlocal stochastic Swift-Hohenberg equation with multiplicative noise is obtained using the Lyapunov-Perron method.

The paper is organized as follows. In Section 2, we recall some definitions and already known results concerning global random attractors. In Section 3, we give uniform a priori estimates of the solution for the nonlocal stochastic Swift-Hohenberg equation with positive kernel, and then a global random attractor for the corresponding random dynamical system is obtained. In Section 4, using the technique of a cut-off function, a global random attractor for nonlocal stochastic Swift-Hohenberg with special non-negative kernel is deduced. In Section 5, we will give the existence of a local random invariant manifold of the corresponding random dynamical system for nonlocal stochastic Swift-Hohenberg equation with multiplicative noise using the Lyapunov-Perron's method.

\section{Preliminaries}

We give some basic concepts related to random attractors and random invariant manifolds. The considered problem for a nonlocal stochastic Swift-Hohenberg equation with multiplicative noise [9] is rewritten as

$$
u_{t}=A u+F(u)+\sigma u \circ \dot{W},
$$


which is subject to initial conditions and boundary conditions as (1.8) and (1.9) on the bounded domain $D \in \mathbb{R}^{2}$, where $A=-\left(1+\partial_{x x}\right)^{2}+\mu$ is a closed self-adjoint linear operator with dense domain $D(A)$ in $H=L^{2}(D), F(u)=-u G * u^{2}$, and $\sigma, \mu>0$ are positive parameters, $W(t)$ is a standard real valued one-dimensional Brownian motion. Assume $H=L^{2}(D)$ with norm $\|\cdot\|$ and scalar product $\langle\cdot, \cdot\rangle$, a real separable Hilbert space.

The eigenvalues of $A$ are defined as $\lambda_{n}(n=1,2, \ldots)$ and the corresponding eigenvectors are $e_{n}(n=1,2, \ldots)$. Let $H_{c}=\operatorname{span}\left\{e_{1}, \ldots, e_{m}\right\}$, and $H_{s}=H \backslash H_{c}$, where $m \in \mathbb{N}$ is finite. Denote the orthogonal projection from $H$ to $H_{c}$ by $P_{c}$ and $A_{c}=P_{c} A$. In addition, denote $P_{s}=I-P_{c}$ and $A_{s}=P_{s} A$. In the following, the subscript ' $c$ ' or ' $s$ ' will represent the projection onto $H_{c}$ or the projection onto $H_{s}$, respectively. We know $P_{c}$ is a continuous projection and can be commuted with $A$ and is not self-adjoint.

Now we give the interpolation spaces $H^{\alpha}$ with $\alpha \in[0,1)$, which are defined as the domain of $A^{\alpha}$ endowed with scalar product $\langle u, v\rangle_{\alpha}=\left\langle A^{\alpha} u, A^{\alpha} v\right\rangle$ and the norm $\|\cdot\|_{\alpha}$. In addition, we define $H^{-\alpha}$ as the dual of $H^{\alpha}$ with respect to the inner product in $H$. For the nonlinear function $F(u)$, which is defined by a cubic function, $F: H^{\alpha} \times H^{\alpha} \times H^{\alpha} \rightarrow H$. We can obtain the local Lipschitz condition by a cut-off function. In addition, we know $H=H_{c} \oplus H_{s}$ and $H^{\alpha}=H_{c} \oplus H_{s}^{\alpha}$, where $H_{s}^{\alpha}=H^{\alpha} \cap H_{s}$ with $\alpha \in[0,1)$. The following properties will be used. From [12-14], there exists $M>0$ such that

$$
\begin{aligned}
& \left\|e^{A t} P_{c}\right\|_{L\left(H^{\alpha}, H^{\alpha}\right)} \leq M e^{\lambda_{c} t}, \quad t \leq 0, \\
& \left\|e^{A t} P_{c}\right\|_{L\left(H^{\alpha}, H\right)} \leq M e^{\lambda_{c} t}, \quad t \leq 0, \\
& \left\|e^{A t} P_{s}\right\|_{L\left(H^{\alpha}, H^{\alpha}\right)} \leq M e^{\lambda_{s} t}, \quad t \geq 0, \\
& \left\|e^{A t} P_{s}\right\|_{L\left(H^{\alpha}, H\right)} \leq \frac{M}{t^{\alpha}} e^{\lambda_{s} t}, \quad t \geq 0,
\end{aligned}
$$

where $\lambda_{c}=\min \left\{e_{1}, \ldots, e_{m}\right\}$ and $\lambda_{s}=\max \left\{e_{m+1}, \ldots\right\}$.

Some concepts of a global random attractor for continuous random dynamical system can be found in [15-20]. Let $\left(X,\|\cdot\|_{X}\right)$ be a separable Hilbert space with Borel $\sigma$-algebra $\mathscr{B}(X)$, and $\left(\Omega, \mathcal{F}, \mathbb{P},\left(\theta_{t}\right)_{t \in \mathbb{R}}\right)$ be an ergodic metric dynamical system. Here, the global random attractor should be considered on a tempered random set.

Definition 2.1 A random set $\{\mathscr{D}(\omega)\}$ is called tempered if for all $\beta>0$ and $\mathbb{P}$-a.e. $\omega \in \Omega$,

$$
\lim _{t \rightarrow+\infty} e^{-\beta t} d\left(\mathscr{D}\left(\theta_{-t} \omega\right)\right)=0
$$

where $d\left(\mathscr{D}\left(\theta_{-t} \omega\right)\right)=\sup \left\{\|b\|_{X}: b \in \mathscr{D}\right\}$.

In order to obtain the main result as regards a global random attractor, only if the conditions of the following theorem obtain, the result of a global random attractor will be given easily and directly.

Theorem 2.1 (See $[17,18])$ Let $\varphi$ be a continuous random dynamical system on $X$ over $\left(\Omega, \mathcal{F}, \mathbb{P},\left(\theta_{t}\right)_{t \in \mathbb{R}}\right)$. If there is a closed random tempered absorbing set $\{B(\omega)\}$ of $\varphi$ and $\varphi$ is 
asymptotically compact in $X$, then $\{A(\omega)\}$ is a random attractor of $\varphi$, where

$$
A(\omega)=\bigcap_{t>0} \overline{\bigcup_{\tau \geq t} \varphi\left(\tau, \theta_{-\tau} \omega, B\left(\theta_{-\tau} \omega\right)\right)}, \quad \omega \in \Omega .
$$

Moreover, $\{A(\omega)\}$ is a unique random attractor of $\varphi$.

Furthermore, the random invariant manifolds of the corresponding random dynamical system will also investigated. The Sobolev spaces $H^{m}(D)$ or $H^{\alpha}(D)$ are the usual Sobolev spaces or fractional Sobolev spaces, for all $m \geq 1$ or $0 \leq \alpha<1$, respectively. The main step is to use the transformation process for our goal, which is given in the following. We consider the scalar Langevin equation

$$
d z+z d t=\sigma d W
$$

It is well known that this equation has a unique stationary solution $z\left(\theta_{t} \omega\right)$, which is called the stationary Ornstein-Uhlenbeck process. The main properties of the stationary Ornstein-Uhlenbeck process $z(t)$ are in particular recalled. Here, set $z(t)=z\left(\theta_{t} \omega\right)$ and $z(0)=z(\omega)$.

Lemma 2.1 (See $[15,16,21-24])$ There exists a subset $\Omega^{*}$ of $\Omega$ which is of full measure and is $\theta_{t}$-invariant for all $t \in \mathbb{R}$, i.e.,

$$
\mathbb{P}\left(\Omega^{*}\right)=1, \quad \theta_{t}\left(\Omega^{*}\right)=\Omega^{*}, \quad \forall t \in \mathbb{R} ;
$$

and the following properties hold on $\Omega^{*}$.

(i) For each $\omega \in \Omega^{*}, t \mapsto W_{t}(\omega)$ is $\gamma$-Hölder for any $\gamma \in\left(0, \frac{1}{2}\right)$.

(ii) $t \mapsto \omega(t)$ has sublinear growth

$$
\lim _{t \rightarrow \pm \infty} \frac{W_{t}(\omega)}{t}=0, \quad \forall \omega \in \Omega^{*}
$$

(iii) For each $\omega \in \Omega^{*}, t \mapsto z\left(\theta_{t} \omega\right)$ is $\gamma$-Hölder for any $\gamma \in\left(0, \frac{1}{2}\right)$, and can be written as

$$
\begin{aligned}
z\left(\theta_{t} \omega\right) & =-\sigma \int_{-\infty}^{0} e^{\tau} W_{\tau}\left(\theta_{t} \omega\right) d \tau \\
& =-\sigma \int_{-\infty}^{0} e^{\tau} W_{\tau+t}(\omega) d \tau+\sigma W_{t}(\omega), \quad t \in \mathbb{R}, \omega \in \Omega^{*}
\end{aligned}
$$

(iv) The following growth control relations are satisfied:

$$
\lim _{t \rightarrow \pm \infty} \frac{z\left(\theta_{t} \omega\right)}{t}=0, \quad \lim _{t \rightarrow \pm \infty} \frac{\int_{0}^{t} z\left(\theta_{\tau} \omega\right) d \tau}{t}=0, \quad \forall \omega \in \Omega^{*}
$$

\section{Nonlocal stochastic Swift-Hohenberg equation with positive kernel}

In this section, we mainly show the global random attractor, for the nonlocal stochastic Swift-Hohenberg equation with positive kernel. Note that equation (1.7) can be interpreted as a system of the integral equation

$$
u(t)=u_{0}-\int_{0}^{t}\left(\alpha u+2 \Delta u+\Delta^{2} u+u G * u^{2}\right) d s+\sigma \int_{0}^{t} u(s) \circ d W(s)
$$


where $\alpha=1-\mu$. For the $\mu \leq 0$ case, it is easy to investigate. So in this paper we assume that $\mu>0$, i.e., $\alpha<1$.

Now, we mainly investigate the global random attractor for the stochastic SwiftHohenberg equation (1.7) with positive kernel. Assume that, for every $t \geq 0$ and $(x, y) \in D$, there are

$$
0<b \leq G\left(\sqrt{x^{2}+y^{2}}\right) \leq a \text { and } G, \nabla G, \Delta G \in L^{\infty}
$$

where $a, b>0$ are some positive constants. That is to say, $G>0$ is a positive kernel and $\nabla=\left(\partial_{x}, \partial_{y}\right)$ is the gradient operator. We denote $K_{1}=\|\nabla G\|_{\infty}$ and $K_{2}=\|\Delta G\|_{\infty}$. These conditions hold throughout.

Set $v=e^{-z\left(\theta_{t}(\omega)\right.} u(t)$. Then from (1.7)-(1.9) we can obtain the following system:

$$
\begin{aligned}
& \frac{d v}{d t}+\left(\alpha-z\left(\theta_{t} \omega\right)\right) v+2 \Delta v+\Delta^{2} v+e^{2 z\left(\theta_{t} \omega\right)} v G * v^{2}=0, \\
& v(x, y, 0)=e^{-z(\omega)} u_{0}, \quad(x, y) \in D, \\
& \left.v\right|_{\partial D}=\left.\frac{\partial v}{\partial n}\right|_{\partial D}=0 .
\end{aligned}
$$

We can use the standard Galerkin method [14, 25] to show that the system (3.2)-(3.4) has a unique solution $v\left(t, \omega, v_{0}\right)$ which is continuous with respect to $v_{0}$ in $H_{0}^{2}(D)$ for all $t>0$ [26]. Then the system (3.2)-(3.4) generates a continuous random dynamical system $\{\varphi(t)\}_{t \geq 0}$ over $\left(\Omega, \mathcal{F}, \mathbb{P},\left(\theta_{t}\right)_{t \in \mathbb{R}}\right)$, where

$$
\varphi\left(t, \omega, v_{0}\right)=v\left(t, \omega, v_{0}\right)
$$

for all $v_{0} \in L^{2}(D), t \geq 0$, and $\omega \in \Omega$. On defining a mapping

$$
\phi\left(t, \omega, v_{0}\right)=u\left(t, \omega, v_{0}\right)=e^{z\left(\theta_{t} \omega\right)} v\left(t, \omega, v_{0}\right)=e^{z\left(\theta_{t} \omega\right)} \varphi\left(t, \omega, v_{0}\right),
$$

we deduce that $\phi$ is a continuous random dynamical system associated with the system (1.7)-(1.9) on $H_{0}^{2}(D)$. Therefore, it is easy to deduce that $\phi$ has a global random attractor only if $\varphi$ possesses a global random attractor. Then we consider the random dynamical system $\varphi$ as follows on the basis of the following uniform a priori estimates of the solution for the system (3.2)-(3.4).

\subsection{Uniform a priori estimates of solution}

In the following, we set $\mathscr{D}$ for the collection of all tempered random subsets in $L^{2}(D)$. First of all, we give some uniform estimates of the solution for the system (3.2)-(3.4) on $D$ when $t \rightarrow \infty$. These estimates are important to obtain the random absorbing set when time is sufficiently large. Now we give some lemmas to show that the random dynamical system $\varphi$ has a random absorbing set in $\mathscr{D}$.

Lemma 3.1 Assume that any $v_{0} \in B(\omega) \subset \mathscr{D}$. Then there exist a $t_{1 B}(\omega)>0$ and a random variable $\rho_{1}(\omega)>0$ such that, for all $\mathbb{P}$-a.e. $\omega \in \Omega$ and $t>t_{1 B}(\omega)$, we have

$$
\left\|v\left(t, \theta_{-t} \omega, v_{0}\left(\theta_{-t} \omega\right)\right)\right\|^{2} \leq \rho_{1}(\omega) .
$$


Proof Taking the inner product with $v(t)$ on the both sides of (3.2) in $D$, then we have

$$
\frac{1}{2} \frac{d}{d t}\|v\|^{2}+\left(\alpha-z\left(\theta_{t} \omega\right)\right)\|v(t)\|^{2}+\langle 2 \Delta v, v\rangle+\|\Delta v\|^{2}+\left\langle e^{2 z\left(\theta_{t} \omega\right)} v G * v^{2}, v\right\rangle=0
$$

Notice

$$
|\langle 2 \Delta v, v\rangle| \leq \frac{1}{2}\|\Delta v\|^{2}+2\|v\|^{2}
$$

In addition, we have

$$
\left\langle e^{2 z\left(\theta_{t} \omega\right)} v G * v^{2}, v\right\rangle=e^{2 z\left(\theta_{t} \omega\right)} \int_{D} v^{2} G * v^{2} d x \geq b e^{2 z\left(\theta_{t} \omega\right)}\|v\|^{4}
$$

Then substituting (3.7) and (3.8) into (3.6), we can obtain

$$
\frac{1}{2} \frac{d}{d t}\|v\|^{2}+\left(\alpha-z\left(\theta_{t} \omega\right)\right)\|v(t)\|^{2}-\frac{1}{2}\|\Delta v\|^{2}-2\|v\|^{2}+\|\Delta v\|^{2}+b e^{2 z\left(\theta_{t} \omega\right)}\|v\|^{4} \leq 0
$$

That is,

$$
\begin{aligned}
& \frac{d}{d t}\|v\|^{2}+2\left(\varepsilon-z\left(\theta_{t} \omega\right)\right)\|v(t)\|^{2}+\|\Delta v\|^{2} \\
& \quad+2(\alpha-2-\varepsilon)\|v(t)\|^{2}+2 b e^{2 z\left(\theta_{t} \omega\right)}\|v\|^{4} \leq 0 .
\end{aligned}
$$

Since $\alpha-1<0, \alpha-2-\varepsilon<0$, and

$$
\begin{aligned}
& 2 b e^{2 z\left(\theta_{t} \omega\right)}\|v\|^{4}+2(\alpha-2-\varepsilon)\|v(t)\|^{2} \\
& \quad=2 b e^{2 z\left(\theta_{t} \omega\right)}\left[\|v\|^{2}+\frac{\alpha-2-\varepsilon}{2 b} e^{-2 z\left(\theta_{t} \omega\right)}\right]^{2}-\frac{(\alpha-2-\varepsilon)^{2}}{2 b} e^{-2 z\left(\theta_{t} \omega\right)},
\end{aligned}
$$

from (3.10), we obtain

$$
\frac{d}{d t}\|v\|^{2}+2\left(\varepsilon-z\left(\theta_{t} \omega\right)\right)\|v(t)\|^{2}+\|\Delta v\|^{2} \leq \frac{(\alpha-2-\varepsilon)^{2}}{2 b} e^{-2 z\left(\theta_{t} \omega\right)}
$$

Now we consider the inequality (3.11). Multiplying $e^{2 \varepsilon t-\int_{0}^{t} 2 z\left(\theta_{\tau} \omega\right) d \tau}$ on both sides of (3.11), we have

$$
\frac{d}{d t}\left[e^{2 \varepsilon t-\int_{0}^{t} 2 z\left(\theta_{\tau} \omega\right) d \tau}\|v\|^{2}\right] \leq \frac{(\alpha-2-\varepsilon)^{2}}{2 b} e^{-2 z\left(\theta_{t} \omega\right)+2 \varepsilon t-\int_{0}^{t} 2 z\left(\theta_{\tau} \omega\right) d \tau}
$$

Taking the integral from 0 to $t$ with respect to $t$ for (3.12), we can obtain

$$
\begin{aligned}
& \left\|v\left(t, \omega, v_{0}(\omega)\right)\right\|^{2} \\
& \quad \leq e^{-2 \varepsilon t+\int_{0}^{t} 2 z\left(\theta_{\tau} \omega\right) d \tau}\left\|v_{0}(\omega)\right\|^{2}+\frac{(\alpha-2-\varepsilon)^{2}}{2 b} \int_{0}^{t} e^{-2 z\left(\theta_{s} \omega\right)-2 \varepsilon(t-s)+\int_{s}^{t} 2 z\left(\theta_{\tau} \omega\right) d \tau} d s .
\end{aligned}
$$

In the above inequality (3.13), replacing $\omega$ by $\theta_{-t} \omega$, we obtain 


$$
\begin{aligned}
& \left\|v\left(t, \theta_{-t} \omega, v_{0}\left(\theta_{-t} \omega\right)\right)\right\|^{2} \\
& \quad \leq e^{-2 \varepsilon t+\int_{0}^{t} 2 z\left(\theta_{\tau-t} \omega\right) d \tau}\left\|v_{0}\left(\theta_{-t} \omega\right)\right\|^{2}+\frac{(\alpha-2-\varepsilon)^{2}}{2 b} \int_{0}^{t} e^{-2 z\left(\theta_{s-t} \omega\right)-2 \varepsilon(t-s)+\int_{s}^{t} 2 z\left(\theta_{\tau}-t \omega\right) d \tau} d s \\
& \quad=e^{-2 t\left(\varepsilon-\frac{\int_{-t}^{0} 2 z\left(\theta_{\tau} \omega\right) d \tau}{t}\right)}\left\|v_{0}\left(\theta_{-t} \omega\right)\right\|^{2}+\frac{(\alpha-2-\varepsilon)^{2}}{2 b} \int_{-t}^{0} e^{2 s\left(\varepsilon-\frac{z\left(\theta_{s} \omega\right)}{s}+\frac{\int_{s}^{0} 2 z\left(\theta_{\tau} \omega\right) d \tau}{-s}\right)} d s \\
& \quad \leq e^{-2 t\left(\varepsilon-\frac{\int_{-t}^{0} 2 z\left(\theta_{\tau} \omega\right) d \tau}{t}\right)}\left\|v_{0}\left(\theta_{-t} \omega\right)\right\|^{2}+\frac{(\alpha-2-\varepsilon)^{2}}{2 b} \int_{-\infty}^{0} e^{2 s\left(\varepsilon-\frac{z\left(\theta_{s} \omega\right)}{s}+\frac{\int_{s}^{0} 2 z\left(\theta_{\tau} \omega\right) d \tau}{-s}\right)} d s .
\end{aligned}
$$

According to the properties of $z\left(\theta_{t} \omega\right)$ and the tempered random set $\mathscr{D}$, there exist a $t_{1 B}(\omega)>$ 0 and a random variable $\rho_{1}(\omega)$ such that, for all $\mathbb{P}$-a.e. $\omega \in \Omega$ and $t>t_{1 B}(\omega)$, there is

$$
\left\|v\left(t, \theta_{-t} \omega, v_{0}\left(\theta_{-t} \omega\right)\right)\right\|^{2} \leq \rho_{1}(\omega)
$$

Then the lemma is completed.

Lemma 3.2 Assume that any $v_{0} \in B(\omega) \subset \mathscr{D}$. Then there exist a $t_{2 B}(\omega)>0$ and a random variable $\rho_{2}(\omega)>0$ such that, for all $\mathbb{P}$-a.e. $\omega \in \Omega$ and $t>t_{2 B}(\omega)$, we have

$$
\int_{t}^{t+1}\left\|\Delta v\left(s, \theta_{-t-1} \omega, v_{0}\left(\theta_{-t-1} \omega\right)\right)\right\|^{2} d s \leq \rho_{2}(\omega)
$$

Proof Considering inequality (3.11) again, we have

$$
\frac{d}{d t}\left[e^{2 \varepsilon t-\int_{0}^{t} 2 z\left(\theta_{\tau} \omega\right) d \tau}\|v\|^{2}\right]+e^{2 \varepsilon t-\int_{0}^{t} 2 z\left(\theta_{\tau} \omega\right) d \tau}\|\Delta v\|^{2} \leq \frac{(\alpha-2-\varepsilon)^{2}}{2 b} e^{-2 z\left(\theta_{t} \omega\right)+2 \varepsilon t-\int_{0}^{t} 2 z\left(\theta_{\tau} \omega\right) d \tau}
$$

Taking the integral from $\hat{T}$ to $t$ with respect to $t$ for the above inequality, where $\hat{T} \leq t$, we obtain

$$
\begin{aligned}
\int_{\hat{T}}^{t} e^{-2 \varepsilon(t-s)+\int_{s}^{t} 2 z\left(\theta_{\tau} \omega\right) d \tau}\|\Delta v\|^{2} d s \\
\leq e^{-2 \varepsilon(t-\hat{T})+\int_{\hat{T}}^{t} 2 z\left(\theta_{\tau} \omega\right) d \tau}\left\|v\left(\hat{T}, \omega, v_{0}(\omega)\right)\right\|^{2} \\
\quad+\frac{(\alpha-2-\varepsilon)^{2}}{2 b} \int_{\hat{T}}^{t} e^{-2 z\left(\theta_{s} \omega\right)-2 \varepsilon(t-s)+\int_{s}^{t} 2 z\left(\theta_{\tau} \omega\right) d \tau} d s .
\end{aligned}
$$

Replacing $t$ by $\hat{T}$ in (3.13), and substituting into the above inequality (3.16), we obtain

$$
\begin{aligned}
& \int_{\hat{T}}^{t} e^{-2 \varepsilon(t-s)+\int_{s}^{t} 2 z\left(\theta_{\tau} \omega\right) d \tau}\left\|\Delta v\left(s, \omega, v_{0}(\omega)\right)\right\|^{2} d s \\
& \quad \leq e^{-2 \varepsilon t+\int_{0}^{t} 2 z\left(\theta_{\tau} \omega\right) d \tau}\left\|v_{0}(\omega)\right\|^{2}+\frac{(\alpha-2-\varepsilon)^{2}}{2 b} \int_{0}^{t} e^{-2 z\left(\theta_{s} \omega\right)-2 \varepsilon(t-s)+\int_{s}^{t} 2 z\left(\theta_{\tau} \omega\right) d \tau} d s .
\end{aligned}
$$

In the above inequality (3.17), replacing $\omega$ by $\theta_{-t} \omega$, we obtain

$$
\begin{aligned}
& \int_{\hat{T}}^{t} e^{-2 \varepsilon(t-s)+\int_{s}^{t} 2 z\left(\theta_{\tau-t} \omega\right) d \tau}\left\|\Delta v\left(s, \theta_{-t} \omega, v_{0}\left(\theta_{-t} \omega\right)\right)\right\|^{2} d s \\
& \quad \leq e^{-2 \varepsilon t+\int_{0}^{t} 2 z\left(\theta_{\tau-t} \omega\right) d \tau}\left\|v_{0}\left(\theta_{-t} \omega\right)\right\|^{2}+\frac{(\alpha-2-\varepsilon)^{2}}{2 b} \int_{0}^{t} e^{-2 z\left(\theta_{s-t} \omega\right)-2 \varepsilon(t-s)+\int_{s}^{t} 2 z\left(\theta_{\tau-t} \omega\right) d \tau} d s .
\end{aligned}
$$


Furthermore, we replace $\hat{T}$ by $t$ and $t$ by $t+1$ in the above inequality. Therefore we obtain

$$
\begin{aligned}
\int_{t}^{t+1} & e^{-2 \varepsilon(t+1-s)+\int_{s}^{t+1} 2 z\left(\theta_{\tau-t-1} \omega\right) d \tau}\left\|\Delta v\left(s, \theta_{-t-1} \omega, v_{0}\left(\theta_{-t-1} \omega\right)\right)\right\|^{2} d s \\
\leq & e^{-2 \varepsilon(t+1)+\int_{0}^{t+1} 2 z\left(\theta_{\tau-t-1} \omega\right) d \tau}\left\|v_{0}\left(\theta_{-t-1} \omega\right)\right\|^{2} \\
\quad & \quad \frac{(\alpha-2-\varepsilon)^{2}}{2 b} \int_{0}^{t+1} e^{-2 z\left(\theta_{s-t-1} \omega\right)-2 \varepsilon(t+1-s)+\int_{s}^{t+1} 2 z\left(\theta_{\tau-t-1} \omega\right) d \tau} d s
\end{aligned}
$$

That is,

$$
\begin{aligned}
& \int_{t}^{t+1} e^{-2 \varepsilon(t+1-s)+\int_{s-t-1}^{0} 2 z\left(\theta_{\tau} \omega\right) d \tau}\left\|\Delta v\left(s, \theta_{-t-1} \omega, v_{0}\left(\theta_{-t-1} \omega\right)\right)\right\|^{2} d s \\
& \leq e^{-2(t+1)\left(\varepsilon-\frac{\int_{-t-1}^{0} 2 z\left(\theta_{\tau} \omega\right) d \tau}{t+1}\right)}\left\|\nu_{0}\left(\theta_{-t-1} \omega\right)\right\|^{2}+\frac{(\alpha-2-\varepsilon)^{2}}{2 b} \int_{-t-1}^{0} e^{-2 z\left(\theta_{s} \omega\right)+2 \varepsilon s+\int_{s}^{0} 2 z\left(\theta_{\tau} \omega\right) d \tau} d s \\
& \leq e^{-2(t+1)\left(\varepsilon-\frac{\int_{-t-1}^{0} 2 z\left(\theta_{\tau} \omega\right) d \tau}{t+1}\right)}\left\|v_{0}\left(\theta_{-t-1} \omega\right)\right\|^{2} \\
& \quad+\frac{(\alpha-2-\varepsilon)^{2}}{2 b} \int_{\infty}^{0} e^{-2 z\left(\theta_{s} \omega\right)+2 \varepsilon s+\int_{s}^{0} 2 z\left(\theta_{\tau} \omega\right) d \tau} d s .
\end{aligned}
$$

According to the properties of $z\left(\theta_{t} \omega\right)$ and $-1 \leq s-t-1 \leq 0$, we have

$$
\begin{aligned}
& \int_{t}^{t+1} e^{-2 \varepsilon(t+1-s)+\int_{s-t-1}^{0} 2 z\left(\theta_{\tau} \omega\right) d \tau}\left\|\Delta v\left(s, \theta_{-t-1} \omega, v_{0}\left(\theta_{-t-1} \omega\right)\right)\right\|^{2} d s \\
& \quad \geq e^{-2 \varepsilon-2 \max _{-1 \leq \tau \leq 0}\left|z\left(\theta_{\tau} \omega\right)\right|} \int_{t}^{t+1}\left\|\Delta v\left(s, \theta_{-t-1} \omega, v_{0}\left(\theta_{-t-1} \omega\right)\right)\right\|^{2} d s .
\end{aligned}
$$

Then from (3.18) and (3.19), we deduce that there exists a random variable $\rho_{2}(\omega)$ for all $\mathbb{P}$-a.e. $\omega \in \Omega$ such that

$$
\int_{t}^{t+1}\left\|\Delta v\left(s, \theta_{-t-1} \omega, v_{0}\left(\theta_{-t-1} \omega\right)\right)\right\|^{2} d s \leq \rho_{2}(\omega), \quad \text { as } t \rightarrow+\infty .
$$

So the lemma is obtained.

Lemma 3.3 Assume that any $v_{0} \in B(\omega) \subset \mathscr{D}$. Then there exist a $t_{3 B}(\omega)>0$ and a random variable $\rho_{3}(\omega)>0$ such that, for all $\mathbb{P}$-a.e. $\omega \in \Omega$ and $t>t_{3 B}(\omega)$, we have

$$
\left\|\Delta v\left(t, \theta_{-t} \omega, v_{0}\left(\theta_{-t} \omega\right)\right)\right\|^{2} d s \leq \rho_{3}(\omega) .
$$

Proof Taking the inner product with $\Delta^{2} v$ on both sides of (3.2) in $D$, we have

$$
\begin{aligned}
& \frac{1}{2} \frac{d}{d t}\|\Delta v\|^{2}+\left(\alpha-z\left(\theta_{t} \omega\right)\right)\|\Delta v(t)\|^{2}+\left\langle 2 \Delta v, \Delta^{2} v\right\rangle+\left\|\Delta^{2} v\right\|^{2} \\
& \quad+\left\langle e^{2 z\left(\theta_{t}(\omega)\right.} v G * v^{2}, \Delta^{2} v\right\rangle=0
\end{aligned}
$$

Notice

$$
\left|\left\langle 2 \Delta v, \Delta^{2} v\right\rangle\right| \leq \frac{1}{2}\left\|\Delta^{2} v\right\|^{2}+2\|\Delta v\|^{2} .
$$


In addition, we have

$$
\begin{aligned}
\left\langle e^{2 z\left(\theta_{t} \omega\right)} v G * v^{2}, \Delta^{2} v\right\rangle \\
=e^{2 z\left(\theta_{t}(\omega)\right.} \int_{D}\left[\Delta v G * v^{2}+2 \nabla v \nabla G * v^{2}+v \Delta G * v^{2}\right] \Delta v d x \\
\leq a e^{2 z\left(\theta_{t} \omega\right)\|v\|^{2}\|\Delta v\|^{2}}+2 \lambda_{1}^{-\frac{1}{2}}\|\nabla G\|_{L^{\infty}} e^{2 z\left(\theta_{t}(\omega)\right.}\|v\|^{2}\|\Delta v\|^{2} \\
\quad+\lambda_{1}^{-1}\|\Delta G\|_{L^{\infty}} e^{2 z\left(\theta_{t} \omega\right)}\|v\|^{2}\|\Delta v\|^{2} \\
\leq a e^{2 z\left(\theta_{t} \omega\right)\|v\|^{2}\|\Delta v\|^{2}}+2 \lambda_{1}^{-\frac{1}{2}} K_{1} e^{2 z\left(\theta_{t} \omega\right)}\|v\|^{2}\|\Delta v\|^{2}+\lambda_{1}^{-1} K_{2} e^{2 z\left(\theta_{t} \omega\right)}\|v\|^{2}\|\Delta v\|^{2} .
\end{aligned}
$$

Then from (3.22), we obtain

$$
\begin{aligned}
& \frac{d}{d t}\|\Delta v\|^{2}+2\left(\varepsilon-z\left(\theta_{t} \omega\right)\right)\|\Delta v(t)\|^{2} \\
& \quad \leq 2\left(a+2 K_{1} \lambda_{1}^{-\frac{1}{2}}+K_{2} \lambda_{1}^{-1}\right) e^{2 z\left(\theta_{t} \omega\right)}\|v\|^{2}\|\Delta v\|^{2}+2(2-\alpha+\varepsilon)\|\Delta v\|^{2} \\
& \quad \doteq 2 K e^{2 z\left(\theta_{t} \omega\right)}\|v\|^{2}\|\Delta v\|^{2}+2(2-\alpha+\varepsilon)\|\Delta v\|^{2} .
\end{aligned}
$$

Using Gronwall's inequality, we have

$$
\begin{aligned}
\left\|\Delta v\left(t+1, \omega, v_{0}(\omega)\right)\right\|^{2} & \\
\leq & e^{-2 \varepsilon(t+1-s)+\int_{s}^{t+1} 2 z\left(\theta_{\tau} \omega\right) d \tau}\left\|\Delta v\left(s, \omega, v_{0}(\omega)\right)\right\|^{2} \\
& +2 \int_{s}^{t+1}\left[K e^{2 z\left(\theta_{\tau} \omega\right)}\left\|v\left(\tau, \omega, v_{0}(\omega)\right)\right\|^{2}+(2-\alpha+\varepsilon)\right] \\
& \cdot e^{-2 \varepsilon(t+1-\tau)+\int_{\tau}^{t+1} 2 z\left(\theta_{r} \omega\right) d r}\left\|\Delta v\left(\tau, \omega, v_{0}(\omega)\right)\right\|^{2} d \tau,
\end{aligned}
$$

where $t \leq s \leq t+1$. In the above inequality (3.25), taking the integral from $t$ to $t+1$ with respect to $s$, we obtain

$$
\begin{aligned}
& \left\|\Delta v\left(t+1, \omega, v_{0}(\omega)\right)\right\|^{2} \\
& \leq \int_{t}^{t+1} e^{-2 \varepsilon(t+1-s)+\int_{s}^{t+1} 2 z\left(\theta_{\tau} \omega\right) d \tau}\left\|\Delta v\left(s, \omega, v_{0}(\omega)\right)\right\|^{2} d s \\
& \quad+2 \int_{t}^{t+1} \int_{s}^{t+1}\left[K e^{2 z\left(\theta_{\tau} \omega\right)}\left\|v\left(\tau, \omega, v_{0}(\omega)\right)\right\|^{2}+(2-\alpha+\varepsilon)\right] \\
& \quad \cdot e^{-2 \varepsilon(t+1-\tau)+\int_{\tau}^{t+1} 2 z\left(\theta_{r} \omega\right) d r}\left\|\Delta v\left(\tau, \omega, v_{0}(\omega)\right)\right\|^{2} d \tau d s .
\end{aligned}
$$

In (3.13), replacing $t$ by $\tau$ and substituting it into the above inequality (3.26), we obtain

$$
\begin{aligned}
& \left\|\Delta v\left(t+1, \omega, v_{0}(\omega)\right)\right\|^{2} \\
& \quad \leq \int_{t}^{t+1} e^{-2 \varepsilon(t+1-s)+\int_{s}^{t+1} 2 z\left(\theta_{\tau} \omega\right) d \tau}\left\|\Delta v\left(s, \omega, v_{0}(\omega)\right)\right\|^{2} d s \\
& \quad+2 K\left\|v_{0}(\omega)\right\|^{2} \int_{t}^{t+1} \int_{s}^{t+1} e^{2 z\left(\theta_{\tau}(\omega)-2 \varepsilon(t+1)+\int_{0}^{t+1} 2 z\left(\theta_{r}(\omega) d r\right.\right.}\left\|\Delta v\left(\tau, \omega, v_{0}(\omega)\right)\right\|^{2} d \tau d s
\end{aligned}
$$




$$
\begin{aligned}
& +\frac{2 K(\alpha-2-\varepsilon)^{2}}{2 b} \int_{t}^{t+1} \int_{s}^{t+1} \int_{0}^{\tau} e^{2 z\left(\theta_{\tau} \omega\right)-2 z\left(\theta_{r} \omega\right)-2 \varepsilon(t+1-r)+\int_{r}^{t+1} 2 z\left(\theta_{\xi} \omega\right) d \xi} \\
& \cdot\left\|\Delta v\left(\tau, \omega, v_{0}(\omega)\right)\right\|^{2} d r d \tau d s \\
& +\int_{t}^{t+1} \int_{s}^{t+1} 2(2-\alpha+\varepsilon) e^{-2 \varepsilon(t+1-\tau)+\int_{\tau}^{t+1} 2 z\left(\theta_{r} \omega\right) d r}\left\|\Delta v\left(\tau, \omega, v_{0}(\omega)\right)\right\|^{2} d \tau d s .
\end{aligned}
$$

It is easy to obtain from the above inequality (3.27) that

$$
\begin{aligned}
\| \Delta v( & \left.t+1, \omega, v_{0}(\omega)\right) \|^{2} \\
\leq & (5-2 \alpha+2 \varepsilon) \int_{t}^{t+1} e^{-2 \varepsilon(t+1-s)+\int_{s}^{t+1} 2 z\left(\theta_{\tau} \omega\right) d \tau}\left\|\Delta v\left(s, \omega, v_{0}(\omega)\right)\right\|^{2} d s \\
& +2 K\left\|v_{0}(\omega)\right\|^{2} \int_{t}^{t+1} \int_{s}^{t+1} e^{2 z\left(\theta_{\tau} \omega\right)-2 \varepsilon(t+1)+\int_{0}^{t+1} 2 z\left(\theta_{r} \omega\right) d r}\left\|\Delta v\left(\tau, \omega, v_{0}(\omega)\right)\right\|^{2} d \tau d s \\
& +\frac{2 K(\alpha-2-\varepsilon)^{2}}{2 b} \int_{t}^{t+1} \int_{s}^{t+1} \int_{0}^{\tau} e^{2 z\left(\theta_{\tau} \omega\right)-2 z\left(\theta_{r} \omega\right)-2 \varepsilon(t+1-r)+\int_{r}^{t+1} 2 z\left(\theta_{\xi} \omega\right) d \xi} \\
& \cdot\left\|\Delta v\left(\tau, \omega, v_{0}(\omega)\right)\right\|^{2} d r d \tau d s .
\end{aligned}
$$

In the above inequality (3.28), replacing $\omega$ by $\theta_{-t-1} \omega$, we obtain

$$
\begin{aligned}
\| \Delta & v\left(t+1, \theta_{-t-1} \omega, v_{0}\left(\theta_{-t-1} \omega\right)\right) \|^{2} \\
\leq & (5-2 \alpha+2 \varepsilon) \int_{t}^{t+1} e^{-2 \varepsilon(t+1-s)+\int_{s}^{t+1} 2 z\left(\theta_{\tau-t-1} \omega\right) d \tau}\left\|\Delta v\left(s, \theta_{-t-1} \omega, v_{0}\left(\theta_{-t-1} \omega\right)\right)\right\|^{2} d s \\
+ & 2 K\left\|v_{0}\left(\theta_{-t-1} \omega\right)\right\|^{2} \int_{t}^{t+1} \int_{s}^{t+1} e^{2 z\left(\theta_{\tau-t-1} \omega\right)-2 \varepsilon(t+1)+\int_{0}^{t+1} 2 z\left(\theta_{r-t-1} \omega\right) d r} \\
& \cdot\left\|\Delta v\left(\tau, \theta_{-t-1} \omega, v_{0}\left(\theta_{-t-1} \omega\right)\right)\right\|^{2} d \tau d s \\
+ & \frac{2 K(\alpha-2-\varepsilon)^{2}}{2 b} \int_{t}^{t+1} \int_{s}^{t+1} \int_{0}^{\tau} e^{2 z\left(\theta_{\tau-t-1} \omega\right)-2 z\left(\theta_{r-t-1} \omega\right)-2 \varepsilon(t+1-r)+\int_{r}^{t+1} 2 z\left(\theta_{\xi-t-1}(\omega)\right) d \xi} \\
& \cdot\left\|\Delta v\left(\tau, \theta_{-t-1} \omega, v_{0}\left(\theta_{-t-1} \omega\right)\right)\right\|^{2} d r d \tau d s .
\end{aligned}
$$

Now, we estimate each term on the right side of the above inequality. For the first term, we have

$$
\begin{gathered}
(5-2 \alpha+2 \varepsilon) \int_{t}^{t+1} e^{-2 \varepsilon(t+1-s)+\int_{s}^{t+1} 2 z\left(\theta_{\tau-t-1} \omega\right) d \tau}\left\|\Delta v\left(s, \theta_{-t-1} \omega, v_{0}\left(\theta_{-t-1} \omega\right)\right)\right\|^{2} d s \\
\leq(5-2 \alpha+2 \varepsilon) e^{2 \max _{-1 \leq \tau \leq 0} \mid z\left(\theta_{\tau} \omega\right)} \int_{t}^{t+1}\left\|\Delta v\left(s, \theta_{-t-1} \omega, v_{0}\left(\theta_{-t-1} \omega\right)\right)\right\|^{2} d s
\end{gathered}
$$

For the second term, we estimate it as follows:

$$
\begin{aligned}
& 2 K \| v_{0}\left(\theta_{-t-1} \omega\right) \|^{2} \int_{t}^{t+1} \int_{s}^{t+1} e^{2 z\left(\theta_{\tau-t-1} \omega\right)-2 \varepsilon(t+1)+\int_{0}^{t+1} 2 z\left(\theta_{r-t-1} \omega\right) d r} \\
& \cdot\left\|\Delta v\left(\tau, \theta_{-t-1} \omega, v_{0}\left(\theta_{-t-1} \omega\right)\right)\right\|^{2} d \tau d s \\
&=2 K\left\|v_{0}\left(\theta_{-t-1} \omega\right)\right\|^{2} e^{-2(t+1)\left(\varepsilon-\frac{\int_{-t-1}^{0} 2 z\left(\theta_{\tau} \omega\right) d \tau}{t+1}\right)} \int_{t}^{t+1} \int_{s}^{t+1} e^{2 z\left(\theta_{\tau-t-1} \omega\right)}
\end{aligned}
$$




$$
\begin{aligned}
& \left\|\Delta v\left(\tau, \theta_{-t-1} \omega, v_{0}\left(\theta_{-t-1} \omega\right)\right)\right\|^{2} d \tau d s \\
\leq & 2 K\left\|v_{0}\left(\theta_{-t-1} \omega\right)\right\|^{2} e^{-2(t+1)\left(\varepsilon-\frac{\int_{-t-1}^{0} 2 z\left(\theta_{\tau} \omega\right) d \tau}{t+1}\right)} e^{2 \max _{-1 \leq \tau \leq 0}\left|z\left(\theta_{\tau} \omega\right)\right|} \\
& \cdot \int_{t}^{t+1}\left\|\Delta v\left(s, \theta_{-t-1} \omega, v_{0}\left(\theta_{-t-1} \omega\right)\right)\right\|^{2} d s .
\end{aligned}
$$

Then, for the third term, we have the estimate

$$
\begin{aligned}
\frac{2 K(\alpha-2-\varepsilon)^{2}}{2 b} \int_{t}^{t+1} \int_{s}^{t+1} \int_{0}^{\tau} e^{2 z\left(\theta_{\tau-t-1} \omega\right)-2 z\left(\theta_{r-t-1} \omega\right)-2 \varepsilon(t+1-r)+\int_{r}^{t+1} 2 z\left(\theta_{\xi-t-1} \omega\right) d \xi} \\
\cdot\left\|\Delta v\left(\tau, \theta_{-t-1} \omega, v_{0}\left(\theta_{-t-1} \omega\right)\right)\right\|^{2} d r d \tau d s \\
\leq \frac{2 K(\alpha-2-\varepsilon)^{2}}{2 b} e^{2 \max _{-1 \leq \tau \leq 0}\left|z\left(\theta_{\tau} \omega\right)\right|} \int_{t}^{t+1} \int_{-t-1}^{\tau-t-1} e^{-2 z\left(\theta_{r} \omega\right)+2 \varepsilon r+\int_{r}^{0} 2 z\left(\theta_{\xi} \omega\right) d \xi} \\
\cdot\left\|\Delta v\left(\tau, \theta_{-t-1} \omega, v_{0}\left(\theta_{-t-1} \omega\right)\right)\right\|^{2} d r d \tau \\
\leq \frac{2 K(\alpha-2-\varepsilon)^{2}}{2 b} e^{2 \max _{-1 \leq \tau \leq 0}\left|z\left(\theta_{\tau} \omega\right)\right|} \int_{t}^{t+1} \int_{-t-1}^{0} e^{2 r\left(\varepsilon-\frac{z\left(\theta_{r} \omega\right)}{r}-\frac{\int_{r}^{0} 2 z\left(\theta_{\xi} \omega\right) d \xi}{-r}\right)} d r \\
\quad \cdot\left\|\Delta v\left(\tau, \theta_{-t-1} \omega, v_{0}\left(\theta_{-t-1} \omega\right)\right)\right\|^{2} d \tau \\
\leq \frac{2 K K_{3}(\alpha-2-\varepsilon)^{2}}{2 b} e^{2 \max _{-1 \leq \tau \leq 0}\left|z\left(\theta_{\tau} \omega\right)\right|} \int_{t}^{t+1}\left\|\Delta v\left(\tau, \theta_{-t-1} \omega, v_{0}\left(\theta_{-t-1} \omega\right)\right)\right\|^{2} d \tau .
\end{aligned}
$$

From the above estimates (3.30), (3.31), and (3.32), we deduce that

$$
\left\|\Delta v\left(t+1, \theta_{-t-1} \omega, v_{0}\left(\theta_{-t-1} \omega\right)\right)\right\|^{2} \leq \rho_{3}(\omega), \quad \text { as } t \rightarrow+\infty
$$

Then the lemma is easy to obtain. The proof is complete.

\subsection{Global random attractor}

According to the former uniform a priori estimates and the lemmas, it is easy to see that there exists a random absorbing set for the random dynamical system generated by (3.2)(3.4) with positive kernel in $H_{0}^{2}(D)$ as the following theorem.

Theorem 3.1 Assume that any $v_{0} \in B(\omega) \subset \mathscr{D}$. Then there exists a random absorbing set $B_{0}(\omega)$ in $H_{0}^{2}(D)$ for the random dynamical system generated by (3.2)-(3.4) with positive kernel in $H_{0}^{2}(D)$.

Proof According to the above three lemmas, we can take $T=\max \left\{T_{1 B}, T_{2 B}, T_{3 B},\right\}$ and $\rho(\omega)=\max \left\{\rho_{1}(\omega), \rho_{2}(\omega), \rho_{3}(\omega)\right\}$. Then, for all $t \geq T$ and $\mathbb{P}$-a.e. $\omega \in \Omega$, there exists a random absorbing set $B_{0}(\omega)$ for the random dynamical system generated by the system (3.2)-(3.4) with positive kernel in $H_{0}^{2}(D)$.

Then according to Theorem 2.1, we know the conditions of Theorem 2.1 have been satisfied. So we easily find the following main result.

Theorem 3.2 Assume that any $v_{0} \in B(\omega) \subset \mathscr{D}$. Then there is a global random attractor in $H_{0}^{2}(D)$ for the random dynamical system generated by the system (3.2)-(3.4) with positive 
kernel. That is to say, the global random attractor is the $\omega$-limit set of the random absorbing set $B_{0}(\omega)$ in $H_{0}^{2}(D)$.

\section{Global random attractor for a nonlocal stochastic Swift-Hohenberg equation with non-negative kernel}

The analysis for positive kernels in Section 3 does not work for non-negative kernels. But we can also obtain some estimates for a special non-negative kernel $G \geq 0$ on the nonlocal stochastic Swift-Hohenberg model. We consider the problem via a mollifier.

Define

$$
J(r)= \begin{cases}c \exp \left(-\frac{1}{1-r^{2}}\right), & r<1, \\ 0, & r \geq 1,\end{cases}
$$

where

$$
c=\left(\int_{D_{1}} \exp \left(-\frac{1}{1-r^{2}}\right) d x\right)^{-1},
$$

$r=\sqrt{x_{1}^{2}+x_{2}^{2}}$, and $D_{1}=\left\{\left(x_{1}, x_{2}\right) \in \mathbb{R}^{2} ; r<1\right\} \subset D$. We also define, for some $\delta>0$,

$$
J_{\delta}(r)=\delta^{-2} J\left(\frac{r}{\delta}\right)
$$

Let $C_{0}(\bar{D})$ be the space of continuous functions with compact support in $D$. Then, for any $f \in C_{0}(\bar{D})$, we define the mollifier of $f\left(y_{1}, y_{2}\right)$ as in [27] by the convolution

$$
J_{\delta} * f=\int_{D} J_{\delta}\left(\sqrt{\left(x_{1}-y_{1}\right)^{2}+\left(x_{2}-y_{2}\right)^{2}}\right) f\left(y_{1}, y_{2}\right) d y_{1} d y_{2} .
$$

It is well known that

$$
\left\|J_{\delta} * f-f\right\|_{C_{0}(\bar{D})} \rightarrow 0 \quad \text { as } \delta \rightarrow 0 .
$$

Therefore, for any given $\varepsilon_{1}>0$, there is a $\delta_{0}=\delta_{0}\left(\varepsilon_{1}\right)>0$ such that

$$
J_{\delta} * f \geq f-\varepsilon_{1} .
$$

We consider the special kernel $G(r)=J_{\delta_{0}}(r)$ with the same family of $\delta_{0}=\delta_{0}\left(\varepsilon_{1}\right)$ as in the above inequality for the stochastic nonlocal Swift-Hohenberg equation. Then $G$ satisfies

$$
0 \leq G \leq \frac{c}{\delta_{0}^{2}}, \quad \nabla G, \Delta G \in L^{\infty}(D)
$$

Notice that the lower bound of $G$ is $b=0$. Then the investigations are different from before. According to the properties above, we can still obtain similar uniform a priori estimates for the nonlocal stochastic Swift-Hohenberg equation. The existence and uniqueness of the solution in $H_{0}^{2}(D)$ for the nonlocal stochastic Swift-Hohenberg equation with 
special kernel $G(r)=J_{\delta_{0}}(r)$ can be obtained as in [14]. Due to the standard regularity argument in $[12,13]$ with the Sobolev embedding theorem, we also have

$$
v(t) \in C_{0}(\bar{D}), \quad \forall b \geq t_{0}>0
$$

Thus implies that

$$
G(r) * v^{2} \geq v^{2}-\varepsilon_{1}
$$

Then we have the following uniform a priori estimates for the nonlocal stochastic SwiftHohenberg equation with the special non-negative kernel $G \geq 0$ in $H_{0}^{2}(D)$.

Lemma 4.1 Assume that any $v_{0} \in B(\omega) \subset \mathscr{D}$. Then there exist a $t_{4 B}(\omega)>0$ and a random variable $\rho_{4}(\omega)>0$ such that, for all $\mathbb{P}$-a.e. $\omega \in \Omega$ and $t>t_{4 B}(\omega)$, there are

$$
\begin{aligned}
& \left\|v\left(t, \theta_{-t} \omega, v_{0}\left(\theta_{-t} \omega\right)\right)\right\|^{2} \leq \rho_{4}(\omega), \\
& \int_{t}^{t+1}\left\|\Delta v\left(s, \theta_{-t-1} \omega, v_{0}\left(\theta_{-t-1} \omega\right)\right)\right\|^{2} d s \leq \rho_{4}(\omega) . \\
& \left\|\Delta v\left(t, \theta_{-t} \omega, v_{0}\left(\theta_{-t} \omega\right)\right)\right\|^{2} \leq \rho_{4}(\omega) .
\end{aligned}
$$

Proof Taking the inner product with $v(t)$ on both sides of (3.2) in $D$, we have

$$
\frac{1}{2} \frac{d}{d t}\|v\|^{2}+\left(\alpha-z\left(\theta_{t} \omega\right)\right)\|v(t)\|^{2}+\langle 2 \Delta v, v\rangle+\|\Delta v\|^{2}+\left\langle e^{2 z\left(\theta_{t} \omega\right)} v G * v^{2}, v\right\rangle=0 .
$$

Notice

$$
|\langle 2 \Delta v, v\rangle| \leq \frac{1}{2}\|\Delta v\|^{2}+2\|v\|^{2} .
$$

In addition, we have

$$
\left\langle e^{2 z\left(\theta_{t} \omega\right)} v G * v^{2}, \nu\right\rangle=e^{2 z\left(\theta_{t} \omega\right)} \int_{D} v^{2} G * v^{2} d x \geq e^{2 z\left(\theta_{t} \omega\right)} \int_{D} \nu^{4} d x d y-\varepsilon_{1} e^{2 z\left(\theta_{t} \omega\right)}\|v\|^{2} .
$$

Then substituting (4.11) and (4.12) into (4.10), we obtain

$$
\begin{aligned}
& \frac{d}{d t}\|v\|^{2}+2\left(\varepsilon-z\left(\theta_{t} \omega\right)\right)\|v(t)\|^{2}+\|\Delta v\|^{2} \\
& \quad \leq \frac{|D|}{4}\left(2(2-\alpha+\varepsilon) \varepsilon_{1}+(2-\alpha+\varepsilon)^{2} e^{-2 z\left(\theta_{t} \omega\right)}+\varepsilon_{1}^{2} e^{2 z\left(\theta_{t} \omega\right)}\right) .
\end{aligned}
$$

When $\varepsilon$ is taken as a sufficiently small positive constant, then we have $2-\alpha+\varepsilon>0$. Therefore, we use similar techniques of estimates to the former, and we obtain the corresponding uniform a priori estimates. So the results of this lemma can be obtained. Here we omit the process.

According to the above lemma, we can obtain the existence of the global random attractor for the nonlocal stochastic Swift-Hohenberg equation with a special non-negative kernel $G$. 
Theorem 4.1 Assume that any $v_{0} \in B(\omega) \subset \mathscr{D}$. Then there is a global random attractor in $H_{0}^{2}(D)$ for the random dynamical system generated by the system (3.2)-(3.4) with a special non-negative kernel G.

From the above investigations and deduction, we can obtain results similar to above for the three-dimensional nonlocal stochastic Swift-Hohenberg equation with the corresponding random dynamical system. Here we do not give the repeated proof and the results. Interested readers can verify all this through similar methods and techniques.

\section{Existence of random invariant manifolds}

\subsection{Transformation and preparations}

Similarly, let $v=e^{-z\left(\theta_{t} \omega\right)} u(t)=e^{-z(t)} u(t)$. Then under the transformation of the OrnsteinUhlenbeck process, the original system (2.1) becomes a random system as follows:

$$
\begin{aligned}
& \frac{d v}{d t}=A v+z v+e^{-z\left(\theta_{t}(\omega)\right.} F\left(v e^{z\left(\theta_{t} \omega\right)}\right), \\
& v(x, y, 0)=e^{-z(\omega)} u_{0}(x, y), \quad(x, y) \in D, \\
& \left.v\right|_{\partial D}=\left.\frac{\partial v}{\partial n}\right|_{\partial D}=0 .
\end{aligned}
$$

In order to consider the random invariant manifolds of the above system in a neighborhood of the fixed point $v=0$, we need to use the cut-off technique such that the nonlinear term satisfies the local Lipschitz condition. Now we give a cut-off function,

$$
\chi(v)= \begin{cases}1 & \text { if }\|v\|_{\alpha} \leq 1, \\ 0 & \text { if }\|v\|_{\alpha}>2\end{cases}
$$

Then, for any positive parameter $R$, we can define $\chi_{R}(v)=\chi\left(\frac{v}{R}\right)$ for all $v \in H^{\alpha}$. Therefore, we obtain

$$
\chi_{R}(v)=\chi\left(\frac{v}{R}\right)= \begin{cases}1 & \text { if }\|v\|_{\alpha} \leq R \\ 0 & \text { if }\|v\|_{\alpha}>2 R\end{cases}
$$

In addition, set $F^{(R)}(v)=\chi_{R}(v) F(v)$. Then, for every positive constant $L_{R}>0$ and for every $\omega \in \Omega$, there exists a positive random variable $R>0$ such that

$$
\left\|F^{(R)}(v)-F^{(R)}(\tilde{v})\right\| \leq L_{R}\|v-\tilde{v}\|_{\alpha} .
$$

Thus we know the nonlinear term $F(v)$ satisfies the local Lipschitz condition (5.4). Then the truncated system by the cut-off technique

$$
u_{t}=-A u+\mu u+F^{(R)}(u)+\sigma u \circ \dot{W}, \quad u(0, x)=u_{0}(x)
$$

will be considered. So by the Ornstein-Uhlenbeck transformation, we will mainly consider the truncated system as follows:

$$
\frac{d v}{d t}=A v+z v+e^{-z\left(\theta_{t} \omega\right)} F^{(R)}\left(v e^{z\left(\theta_{t} \omega\right)}\right),
$$




$$
\begin{aligned}
& v(x, y, 0)=e^{-z(\omega)} u_{0}(x, y), \quad(x, y) \in D \\
& \left.v\right|_{\partial D}=\left.\frac{\partial v}{\partial n}\right|_{\partial D}=0
\end{aligned}
$$

\subsection{Existence of random invariant manifold}

In the following, we mainly show the random invariant manifolds for the system (5.1)(5.3). First of all, we construct the local random invariant manifold of it for sufficiently small $R>0$ and prove its exponentially attracting properties of the corresponding random dynamical system by using the method in [23]. In order to obtain our goals, we should consider the following two systems:

$$
\begin{array}{ll}
\partial_{t} v_{c}=(A+z) v_{c}+e^{-z} P_{c} F^{(R)}\left(v e^{z}\right), & v_{c}(0)=\xi=P_{c} u_{0} e^{-z(0)}, \\
\partial_{t} v_{s}=(A+z) v_{s}+e^{-z} P_{s} F^{(R)}\left(v e^{z}\right), & v_{s}(0)=P_{s} u_{0} e^{-z(0)}
\end{array}
$$

Since $\mu>0$, for any value of $\mu$, there exists a real number $\eta$ such that $\lambda_{s}<\eta<\lambda_{c}$, where $\lambda_{c}$ and $\lambda_{s}$ are the last eigenvalue and the first eigenvalue for the projectors $P_{c}$ and $P_{s}$, respectively. The Lyapunov-Perron method will be used as in [23] on the random space with random norm depending on $\omega$, which is defined as follows.

Definition 5.1 For $\lambda_{s}<\eta<\lambda_{c}$, define the Banach space

$$
C_{\eta}^{-}=\left\{v \in C\left((-\infty, 0] ; H^{\alpha}\right): \sup _{t \leq 0}\left\{e^{-\eta t-\int_{0}^{t} z(r) d r}\|v\|_{\alpha}\right\}<\infty\right\}
$$

with norm

$$
\|v\|_{C_{\eta}^{-}}=\sup _{t \leq 0}\left\{e^{-\eta t-\int_{0}^{t} z(r) d r}\|v\|_{\alpha}\right\}
$$

Notice that, for fixed $t$, as $r \rightarrow-\infty$, we can obtain the following result:

$$
\begin{aligned}
\left\|e^{A(t-r)+\int_{r}^{t} z(\tau) d \tau} v_{s}(r)\right\|_{\alpha} & \leq M e^{\lambda_{s}(t-r)+\int_{r}^{t} z(\tau) d \tau}\left\|v_{s}(r)\right\|_{\alpha} \\
& \leq M\left\|v_{s}\right\|_{C_{\eta}^{-}} e^{-\left(\lambda_{s}-\eta\right) r} e^{\lambda_{s} t+\int_{0}^{t} z(\tau) d \tau} \rightarrow 0 .
\end{aligned}
$$

Now we give a mapping definition according to (5.9)-(5.10) as follows. One can investigate it by the uniform contraction mapping principle as in [23].

Definition 5.2 Define the nonlinear operator $\mathcal{J}$ on $C_{\eta}^{-}$for given $\xi \in H_{c}^{\alpha}$ and $\omega \in \Omega_{0}$ as

$$
\begin{aligned}
\mathcal{J}(v, \xi)(t)= & e^{A t+\int_{0}^{t} z(\tau) d \tau} \xi+\int_{0}^{t} e^{A(t-s)+\int_{s}^{t} z(\tau) d \tau} e^{-z(s)} P_{c} F^{(R)}\left(v(s) e^{z(s)}\right) d s \\
& +\int_{-\infty}^{t} e^{A(t-s)+\int_{s}^{t} z(\tau) d \tau} e^{-z(s)} P_{s} F^{(R)}\left(e^{z(s)} v(s)\right) d s .
\end{aligned}
$$

According to the Lipschitz property of $F^{(R)}(u)$, it is easy to directly see that, for any $\xi \in$ $H_{c}^{\alpha}$ and $\omega \in \Omega_{0}$, there is a mapping $\mathcal{J}(\cdot, \xi)(t): C_{\eta}^{-} \rightarrow C_{\eta}^{-}$; refer to [25]. Furthermore, we can show it is a Lipschitz continuous map according to some estimates as follows. 
Theorem 5.1 Suppose $\lambda_{s}<\eta<\lambda_{c}$ and we have condition (5.16). Then the operator $\mathcal{J}$ has a unique fixed point $v^{*}=v^{*}(\omega, \xi) \in C_{\eta}^{-}$.

Proof For any $v, \bar{v} \in C_{\eta}^{-}$, one considers the estimate

$$
\begin{aligned}
& \|\mathcal{J}(v, \xi)-\mathcal{J}(\bar{v}, \xi)\|_{C_{\eta}^{-}} \\
& \quad \leq M L_{R}\|v-\tilde{v}\|_{C_{\eta}^{-}} \sup _{t \leq 0} \int_{0}^{t} e^{\left(\lambda_{c}-\eta\right)(t-s)} d s+M L_{R}\|v-\bar{v}\|_{C_{\eta}^{-}} \sup _{t \leq 0} \int_{-\infty}^{t} \frac{e^{\left(\lambda_{s}-\eta\right)(t-s)}}{(t-s)^{\alpha}} d s \\
& \quad \leq M L_{R}\left[\frac{1}{\lambda_{c}-\eta}+\frac{\Gamma(1-\alpha)}{\eta-\lambda_{s}}\right]\|v-\bar{v}\|_{C_{\eta}^{-}} \dot{=} \operatorname{Lip}(\mathcal{J})\|v-\bar{v}\|_{C_{\eta}^{-}},
\end{aligned}
$$

where $\operatorname{Lip}(\mathcal{J})=M L_{R}\left[\frac{1}{\lambda_{c}-\eta}+\frac{\Gamma(1-\alpha)}{\eta-\lambda_{s}}\right]$ is actually independent of $\xi$ and $\omega$. Therefore, if for sufficient small $R>0$ we have

$$
\operatorname{Lip}(\mathcal{J})=M L_{R}\left[\frac{1}{\lambda_{c}-\eta}+\frac{\Gamma(1-\alpha)}{\eta-\lambda_{s}}\right]<1
$$

the mapping $\mathcal{J}(\cdot, \xi)(t): C_{\eta}^{-} \rightarrow C_{\eta}^{-}$is a contraction. So according to the uniform contraction mapping principle, we can obtain the result of the theorem.

Furthermore, we can use a similar method to obtain the estimate

$$
\|\mathcal{J}(v, \xi)-\mathcal{J}(v, \bar{\xi})\|_{C_{\eta}^{-}} \leq \frac{M}{1-M L_{R}\left[\frac{1}{\lambda_{c}-\eta}+\frac{\Gamma(1-\alpha)}{\eta-\lambda_{s}}\right]}\|\xi-\bar{\xi}\|
$$

Only if the condition

$$
\frac{M}{1-M L_{R}\left[\frac{1}{\lambda_{c}-\eta}+\frac{\Gamma(1-\alpha)}{\eta-\lambda_{s}}\right]}<1
$$

is satisfied, then the Lipschitz continuous about $\xi$ for $\mathcal{J}(v, \xi)$ can hold. So, for the fixed point $v^{*}=v^{*}(\omega ; \xi) \in C_{\eta}^{-}$, define $h(\omega, \xi)=P_{s} v^{*}(0, \omega ; \xi)$. Thus, we have

$$
h(\omega, \xi)=\int_{-\infty}^{0} e^{-A s+\int_{s}^{0} z(\tau) d \tau} e^{-z(s)} P_{s} F^{(R)}\left(v^{*}(s, \xi) e^{z(s)}\right) d s
$$

Then by a similar discussion to [24], we have a local random Lipschitz invariant manifold for the random dynamical system $\varphi^{R}(t, \omega)$ of the system (2.1) as follows:

$$
M_{c u t}^{R}(\omega)=\left\{\left(\xi, e^{z(\omega)} h\left(\omega, e^{-z(\omega)} \xi\right)\right): \xi \in H_{c}^{\alpha}\right\}
$$

which is the graph of $e^{z(\omega)} h\left(\xi, e^{-z(\omega)} \xi\right)$. In addition, define a mapping

$$
\psi(\omega, \cdot): H_{c} \cap B_{R}(0) \rightarrow H_{s}^{\alpha}, \quad \xi \rightarrow \psi(\omega, \xi)=e^{z(\omega)} h\left(\xi, e^{-z(\omega)} \xi\right) .
$$

So, it is easy to obtain

$$
\mathcal{M}^{R}(\omega)=\operatorname{graph}(\psi(\omega, \cdot))=\mathcal{M}_{\text {cut }}^{R}(\omega) \cap B_{R}(0),
$$


a local Lipschitz random invariant manifold of the random dynamical system $\varphi(t, \omega)$ for the system (2.1).

Theorem 5.2 Suppose we have $\lambda_{s}<\eta<\lambda_{c}$ and conditions (5.16) and (5.18). Then the random dynamical system $\varphi(t, \omega)$ for the system (2.1) has a local Lipschitz random invariant manifold $\mathcal{M}^{R}(\omega)$ for sufficiently small $R>0$, which is defined as the graph of a random Lipschitz map $h(\omega, \cdot): H_{c} \rightarrow H_{s}^{\alpha}$

$$
M_{c u t}^{R}(\omega)=\left\{\left(\xi, e^{z(\omega)} h\left(\omega, e^{-z(\omega)} \xi\right)\right): \xi \in H_{c}\right\},
$$

where $h(\omega, \xi)$ is represented by (5.19).

\section{Competing interests}

The author declares that they have no competing interests.

\section{Acknowledgements}

This research was financially supported by the project of outstanding young teachers' training in higher education institutions of Guangxi, and by NSFC Grant No. 11301097, Guangxi NSF Grant No. 2013GXNSFAA019001, 2014GXNSFAA118016, Guangxi Scientific Research Item of University No. 2013 YB170 and CSC.

Received: 3 July 2015 Accepted: 6 November 2015 Published online: 21 November 2015

\section{References}

1. Swift, J, Hohenberg, PC: Hydrodynamic fluctuations at the convective instability. Phys. Rev. A 15, 319-328 (1977)

2. Eckmann, JP, Wayne, CE: Propagating fronts and the center manifold theorem. Commun. Math. Phys. 136, 285-307 (1991)

3. Hilali, MF, Metens, S, Borckmans, P, Dewel, G: Pattern selection in the generalized Swift-Hohenberg equation. Phys. Rev. E 51, 2046-2052 (1995)

4. Mielke, A, Schneider, G: Attractors for modulation equations on unbounded domains: existence and comparison. Nonlinearity 8, 734-768 (1995)

5. Hsieh, DY, Tang, AQ, Wang, XP: On hydrodynamics instabilities, chaos, and phase transition. Acta Mech. Sin. 12, 1-14 (1996)

6. Oh, J, Ortiz de Zárate, JM, Sengers, JV, Ahlers, G: Dynamics of fluctuations in a fluid below the onset of Rayleigh-Bénard convection. Phys. Rev. E 69, 021106 (2004)

7. Oh, J, Ahlers, G: Thermal-noise effect on the transition to Rayleigh-Bénard convection. Phys. Rev. Lett. 91, 094501 (2003)

8. Rehberg, I, Rasenat, S, de la Torre Juárez, M, Schöpf, W, Hörner, F, Ahlers, G Brand, HR: Thermally induced hydrodynamic fluctuations below the onset of electroconvection. Phys. Rev. Lett. 67, 596-599 (1991)

9. Blómker, D, Hairer, M, Pavliotis, GA: Stochastic Swift-Hohenberg equation near a change of stability. In: Proceedings of Equadiff, vol. 11, pp. 27-37 (2005)

10. Roberts, AJ: Planform evolution in convection: an embedded center manifold. J. Aust. Math. Soc. Ser. B, Appl. Math 34, 174-198 (1992)

11. Roberts, AJ: The Swift-Hohenberg equation requires nonlocal modifications to model spatial pattern evolution of physical problems. arXiv:patt-sol/9412002

12. Henry, D: Geometric Theory of Semilinear Parabolic Equations. Springer, Berlin (1981)

13. Pazy, A: Semigroup of Linear Operators and Applications to Partial Differential Equations. Springer, Berlin (1983)

14. Temam, R: Infinite-Dimensional Dynamical Systems in Mechanics and Physics. Springer, New York (1988)

15. Arnold, L: Random Dynamical Systems. Springer, New York (1998)

16. Chueshov, I: Monotone Random Systems Theory and Applications. Springer, New York (2002)

17. Crauel, H, Debussche, A, Flandoli, F: Random attractors. J. Dyn. Differ. Equ. 9, 307-341 (1997)

18. Crauel, H, Flandoli, F: Attractors for random dynamical systems. Probab. Theory Relat. Fields 100, 365-393 (1994)

19. Duan, J, Wang, W: Effective Dynamics of Stochastic Partial Differential Equations. Elsevier, London (2014)

20. Wang, ZJ, Zhou, SF: Random attractor for stochastic reaction-diffusion equation with multiplicative noise on unbounded domains. J. Math. Anal. Appl. 384, 160-172 (2011)

21. Bates, PW, Lu, K, Wang, B: Random attractors for stochastic reaction-diffusion equations on unbounded domains. J. Differ. Equ. 246, 845-869 (2009)

22. Chekroun, MD, Liu, HH, Wang, SH: Stochastic Parameterizing Manifolds and Non-Markovian Reduced Equations: Stochastic Manifolds for Nonlinear SPDEs II. Springer Briefs in Mathematics. Springer, New York (2015)

23. Duan, J, Lu, K, Schmalfuss, B: Invariant manifolds for stochastic differential equations. Ann. Probab. 31, 2109-2135 (2003)

24. Fan, X: Attractors for a damped stochastic wave equation of Sine-Gordon type with sublinear multiplicative noise. Stoch. Anal. Appl. 24, 767-793 (2006)

25. Da Prato, G, Zabczyk, J: Stochastic Equations in Infinite Dimensions. Cambridge University Press, New York (1992)

26. Lin, G, Gao, H, Duan, J, Ervin, VJ: Asymptotic dynamical difference between the nonlocal and local Swift-Hohenberg models. J. Math Phys. 41, 2077-2089 (2000)

27. Friedman, A: Partial Differential Equations. Holt, Rinehart \& Winston, New York (1969) 